\title{
Pairs of additive forms and Artin's conjecture
}

by

\author{
H. Godinho (Brasília, DF) and C. Ripoll (Porto Alegre, RS)
}

1. Introduction. Let $(f, g)$ be the pair of additive forms

$$
\begin{aligned}
& f=a_{1} x_{1}^{k}+\ldots+a_{n} x_{n}^{k}, \\
& g=b_{1} x_{1}^{k}+\ldots+b_{n} x_{n}^{k}
\end{aligned}
$$

of degree $k>1$ with coefficients in the rational integers. We are concerned with conditions on the number of indeterminates $n$ which guarantees that (1) is solvable in any $p$-adic field, i.e., that there exists a common nontrivial $p$-adic zero for the pair $(f, g)$. Artin has conjectured that $n \geq 2 k^{2}+1$ can be such a condition. This conjecture was confirmed by Demyanov in the case $k=2$ (see $[\mathrm{D}]$ ) and by Davenport and Lewis in the case $k=3$ (see [DL1]). Both also proved that this bound is the best possible in those cases. Later on, Davenport and Lewis in [DL2] proved that this conjecture is also true in the case of $k$ odd. For $k$ even they proved that (1) is solvable in any $p$-adic field, except maybe for the primes $p$ such that $p(p-1)$ divides $k$, or for $p=5$ when 10 divides $k$ (see [DL2], Sections 6-8, and the proof of Theorem 4). They also remarked that the main obstacles lie in the degrees of the type $k=2^{l} k_{0}$, in these cases they could prove that $n \geq 7 k^{3}$ variables would suffice.

Godinho [G2] improved the condition $n \geq 7 k^{3}$ in the case $k=2^{l}, l \geq 2$ :

TheOREM 1. If $k=2^{l}, l \geq 2$, then (1) is solvable in the field of $p$-adic numbers for any prime $p$ if $n \geq 16 k^{2}-26 k+1$.

In [G3] he proved the following

THEOREM 2. Let $p$ be a prime number. If $k=(p-1) p^{\tau} k_{0}, \tau>0$ and $\left(k_{0}, p\right)=1$, then there exists a solution for (1) in $\mathbb{Q}_{p}$ provided that

1991 Mathematics Subject Classification: 11D79, 11D72.

The authors were partially supported by a grant of FAP/DF.

The case of systems of additive forms is treated in a forthcoming paper by J. Brüdern and H. Godinho. 
$n \geq 2 k^{2+w(\tau, p)}$, where

$$
w(\tau, p)=\frac{1}{\log _{2} p+(1 / \tau) \log _{2}(p-1)} .
$$

Notice that when $p$ is large the bound on $n$ gets closer to Artin's bound.

The purpose of this article is to present a new absolute bound for $n$ which does not depend on the values of $\tau$ and of the prime, and which is closer to Artin's conjecture in the remaining cases (i.e. $k=(p-1) p^{\tau} k_{0}, \tau>0$ and $\left(k_{0}, p\right)=1$ or $\left.k=5^{\tau} 2 k_{0}, \tau \geq 1\right)$.

The main results proved in this paper are:

Theorem A. If $k=2 \cdot 3^{\tau} k_{0}$, where $\left(k_{0}, 3\right)=1, \tau \geq 1$ and $n \geq 8 k^{2}$, then (1) is solvable in the field of 3-adic numbers.

Theorem B. If $n \geq 2 k^{5 / 2}$ then (1) is solvable in $\mathbb{Q}_{p}$ for every prime $p$, provided $k \neq 2^{2}, 2^{3}, 2^{4}, 2^{5}$.

Other interesting aspects of this theory are presented in the paper of Atkinson and Cook (see $[\mathrm{AC}]$ ), where it is shown that if $n>4 k+1$ then solvability in $\mathbb{Q}_{p}$ is guaranteed for every prime $p$ such that $p>k^{6}$, showing that Artin's conjecture can fail only for small primes. For more information on the subject we suggest the papers of Atkinson, Brüdern and Cook [ABC], and Wooley $[\mathrm{W}]$, where the case of unequal degree is considered.

2. Preliminary results. The criterion used here to establish that (1) is solvable in $\mathbb{Q}_{p}$ is stated in the next lemma, also due to Davenport and Lewis. We state it in the way we need:

Lemma 3. Let $(f, g)$ be a pair of forms as in $(1), k=(p-1) p^{\tau} k_{0}$ with $\tau \geq 1$ and $\left(k_{0}, p\right)=1$, and let $\gamma$ be defined as follows:

$$
\gamma= \begin{cases}\tau+1 & \text { if } p>2 \\ \tau+2 & \text { if } p=2\end{cases}
$$

If the system

$$
f \equiv 0\left(\bmod p^{\gamma}\right), \quad g \equiv 0\left(\bmod p^{\gamma}\right)
$$

has a solution $\left(x_{1}, \ldots, x_{n}\right)$ in the rational integers for which the matrix

$$
\left(\begin{array}{ccc}
a_{1} x_{1} & \ldots & a_{n} x_{n} \\
b_{1} x_{1} & \ldots & b_{n} x_{n}
\end{array}\right)
$$

has rank 2 modulo $p\left(i . e .\left(a_{i} b_{j}-a_{j} b_{i}\right) x_{i} x_{j} \not \equiv 0(\bmod p)\right.$ for some $\left.i, j\right)$, then the pair $(f, g)$ admits a nontrivial common p-adic zero.

Proof. See Lemma 7 in [DL2]. 
Definition 1. Let $(f, g)$ be a pair of forms as in (1). Any solution for the congruences

$$
f \equiv 0\left(\bmod p^{i}\right), \quad g \equiv 0\left(\bmod p^{i}\right)
$$

for which the matrix

$$
\left(\begin{array}{lll}
a_{1} x_{1} & \ldots & a_{n} x_{n} \\
b_{1} x_{1} & \ldots & b_{n} x_{n}
\end{array}\right)
$$

has rank 2 modulo $p$, will be called a nonsingular solution (or zero) modulo $p^{i}$.

DeFinition 2. If in the pair of forms $(f, g)$, a pair of subforms

$$
a_{1} x_{1}^{k}+\ldots+a_{\mu} x_{\mu}^{k}, \quad b_{1} x_{1}^{k}+\ldots+b_{\mu} x_{\mu}^{k}
$$

can be found which has a nonsingular zero modulo $p^{i}$, then we will call this pair of subforms a nonsingular set of level $i$ and will denote it by $\mathrm{NS} p^{i}$.

With the above definition we can restate Lemma 3 saying that if we can find an $\mathrm{NS}^{\gamma}$ set then (1) is solvable in $\mathbb{Q}_{p}$.

Definition 3. Consider the system

$$
\left\{\begin{array}{l}
f \equiv 0\left(\bmod p^{t}\right) \\
g \equiv 0\left(\bmod p^{t}\right)
\end{array}\right.
$$

for a fixed power $t$. Any nontrivial solution for the above system, with some variable assuming a value which is co-prime to $p$, will be called a primitive solution modulo $p^{t}$ for the pair $(f, g)$.

TheOREM 4. Let $c_{1}, \ldots, c_{m}, d_{1}, \ldots, d_{m} \in \mathbb{Z}$ where at least one in each pair $c_{i}, d_{i}$ is not divisible by $p$. Let $k=p^{\tau} \delta k_{0},\left(k_{0}, p\right)=1$, and $\delta=(p-1, k)$. If $m \geq 2 \delta+1$ then the system

$$
\begin{aligned}
& F=c_{1} x_{1}^{k}+\ldots+c_{m} x_{m}^{k} \equiv 0(\bmod p), \\
& G=d_{1} x_{1}^{k}+\ldots+d_{m} x_{m}^{k} \equiv 0(\bmod p)
\end{aligned}
$$

admits a primitive solution modulo $p$.

Pr o o f. This is Chevalley's Theorem as described in Lemma 2 in [DL2].

THEOREM 5. With the assumptions of Theorem 4, assume further that every form $\alpha F+\beta G$ with $\alpha, \beta \in \mathbb{Z},(\alpha, \beta) \not \equiv(0,0)(\bmod p)$ contains at least $\delta+1$ variables with coefficients not divisible by $p$. Then (2) has a nonsingular solution modulo $p$ (that is, an $\mathrm{NSp}$ set can be formed).

Proof. This is Lemma 3 in [DL2].

We now observe that given a pair $(f, g)$ as in (1) we can always write it in the form

$$
\begin{aligned}
& f=f_{0}+p f_{1}+\ldots+p^{s} f_{s}, \\
& g=g_{0}+p g_{1}+\ldots+p^{s} g_{s},
\end{aligned}
$$


where $f_{i}, g_{i}$ are also forms of degree $k$ in the variables $x_{j}$ of $(f, g), j=$ $1, \ldots, n$, satisfying the following condition: $x_{j}$ is involved in the pair $\left(f_{j}, g_{j}\right)$ if and only if $i=\min \left(v_{p}\left(a_{j}\right), v_{p}\left(b_{j}\right)\right)$, where $v_{p}$ denotes the $p$-adic valuation.

Definition 4 . We will refer to the decomposition of a pair described above in (2) as the canonical decomposition (or form) of $(f, g)$. Moreover for each $i \in\{0,1, \ldots, s\}$ let $m_{i}$ be the number of variables present in the pair $\left(f_{i}, g_{i}\right)$. If a variable occurs in the pair $\left(f_{i}, g_{i}\right)$ then it will be said to be at level $i$.

DeFinition 5. Let

$$
a_{1} x_{1}^{k}+\ldots+a_{\mu} x_{\mu}^{k}, \quad b_{1} x_{1}^{k}+\ldots+b_{\mu} x_{\mu}^{k}
$$

be a pair of subforms of $(f, g)$, with its variables found among the variables of the first pairs $\left(f_{0}, g_{0}\right), \ldots,\left(f_{j}, g_{j}\right)$ of the canonical decomposition of $(f, g)$, and assume they have a common nontrivial zero $\vec{\xi}=\left(\xi_{1}, \ldots, \xi_{\mu}\right)$ modulo $p^{i}$ for some $i>j$. Multiply $\vec{\xi}$ by a new variable $T$ to have

$$
\begin{aligned}
\left(a_{1} \xi_{1}^{k}+\ldots+a_{\mu} \xi_{\mu}^{k}\right) T^{k} & \equiv p^{i} \alpha T^{k}\left(\bmod p^{i+1}\right), \\
\left(b_{1} \xi_{1}^{k}+\ldots+b_{\mu} \xi_{\mu}^{k}\right) T^{k} & \equiv p^{i} \beta T^{k}\left(\bmod p^{i+1}\right)
\end{aligned}
$$

for some $\alpha, \beta \in \mathbb{Z}$. With this procedure a new variable was created at level $i>j$ or higher, and $\mu$ variables were suppressed from the lower levels. The replacement of $\left(x_{1}, \ldots, x_{\mu}\right)$ by $\left(\xi_{1} T, \ldots, \xi_{\mu} T\right)$ is called contraction of $\mu$ variables at level $j$ to a variable at level $i$ or higher.

REMARK. Since an NSp $p^{i}$ set is a pair of subforms with a nonsingular zero modulo $p^{i}$, it makes sense to speak of contracting an $\mathrm{NS}^{i}$ set to a new variable $T$ at level $i$ or higher, but in this case we are going to assume that the zero used in the contraction procedure described in Definition 5 is the nonsingular zero modulo $p^{i}$, ensured by the definition of an $\mathrm{NS} p^{i}$ set.

3. Congruences modulo powers of $\mathbf{3}$. We will denote by $(\widetilde{f}, \widetilde{g})$ the pair of forms obtained from $(f, g)$ after any process of contraction of variables explained in Definition 5, and will denote by

$$
\widetilde{f}=\widetilde{f}_{0}+p \widetilde{f}_{1}+\ldots+p^{s} \widetilde{f}_{s}, \quad \widetilde{g}=\widetilde{g}_{0}+p \widetilde{g}_{1}+\ldots+p^{s} \widetilde{g}_{s}
$$

the canonical form of the pair $(\widetilde{f}, \widetilde{g})$ and by $\widetilde{m}_{i}$ the number of variables present in the pair $\left(\widetilde{f}_{i}, \widetilde{g}_{i}\right)$.

In order to determine a bound for the number of new variables obtained by contraction of the variables of level 0 , we decompose again the variables involved in the pair $\left(f_{0}, g_{0}\right)$ of the canonical decomposition of $(f, g)$ into subsets $R_{1}, \ldots, R_{w}$ according to the ratios modulo 3 of the coefficients $a_{i} / b_{i}$ of its variables. It is an easy exercise to verify that there are at most 4 different classes modulo 3 , i.e., $w=4$. 
Lemma 6. A single congruence $a_{1} x_{1}^{k}+\ldots+a_{n} x_{n}^{k} \equiv 0(\bmod 3)$ admits a primitive solution modulo 3 if $n \geq 3$.

Proof. If $a_{1}=a_{2}=a_{3}$ then we can choose $x_{1}=x_{2}=x_{3}=1$ and all other variables zero. If not, say $a_{1}=1, a_{2}=2$, then we can choose $x_{1}=1=x_{2}$ and all other variables zero.

Lemma 7. In the pair of forms $(f, g)$ as in (1) we can effectively create $\left[r_{1} / 3\right]+\ldots+\left[r_{4} / 3\right]$ new variables from the variables at level 0 , where $r_{i}$ denotes the cardinality of the set $R_{i}$ defined above. Moreover,

$$
\left[r_{1} / 3\right]+\ldots+\left[r_{4} / 3\right] \geq\left[m_{0} / 3\right]-2 .
$$

Proof. In order to prove the first assertion observe that if $a_{1} / b_{1}, a_{2} / b_{2}$, $a_{3} / b_{3}$ are in the same class then

$$
\left\{\begin{array}{l}
a_{1} x_{1}^{k}+a_{2} x_{2}^{k}+a_{3} x_{3}^{k} \equiv 0(\bmod 3), \\
b_{1} x_{1}^{k}+b_{2} x_{2}^{k}+b_{3} x_{3}^{k} \equiv 0(\bmod 3)
\end{array}\right.
$$

admits a nontrivial solution modulo 3 if and only if $a_{1} x_{1}^{k}+a_{2} x_{2}^{k}+a_{3} x_{3}^{k} \equiv 0$ $(\bmod 3)$ does. But this last condition is true by the above lemma. Hence any three pairs of coefficients which belong to the same class can be contracted to a new variable at level 1 or higher. In this way we can then construct $\left[r_{1} / 3\right]+\ldots+\left[r_{4} / 3\right]$ new variables.

Now denote by $t_{i}$ the number $\left[r_{i} / 3\right]$. Then for each $i$ there exists $s_{i} \in$ $\{0,1,2\}$ such that $r_{i}=3 t_{i}+s_{i}$. Then

$$
\begin{aligned}
m_{0} & =\left(3 t_{1}+s_{1}\right)+\ldots+\left(3 t_{4}+s_{4}\right)=3\left(t_{1}+\ldots+t_{4}\right)+s_{1}+\ldots+s_{4} \\
& \leq 3\left(t_{1}+\ldots+t_{4}\right)+4 \cdot 2=3\left(t_{1}+\ldots+t_{4}+2\right)+2 .
\end{aligned}
$$

Hence

$$
\left[m_{0} / 3\right] \leq t_{1}+\ldots+t_{4}+2 .
$$

The above lemma guarantees that at least $\left[m_{0} / 3\right]-2$ new variables can be obtained by contraction of the variables at level zero. We now turn to congruences modulo $3^{2}$.

Lemma 8. Let $(f, g)$ be a pair of additive forms of degree $k=2 \cdot 3^{\tau} k_{0}$, with $\tau \geq 1,\left(k_{0}, 3\right)=1$. Then the system of congruences

$$
\left\{\begin{array}{l}
f=a_{1} x_{1}^{k}+\ldots+a_{n} x_{n}^{k} \equiv 0\left(\bmod 3^{2}\right) \\
g=b_{1} x_{1}^{k}+\ldots+b_{n} x_{n}^{k} \equiv 0\left(\bmod 3^{2}\right)
\end{array}\right.
$$

admits a primitive solution modulo $3^{2}$ if $n \geq 21$.

Proof. Let

$$
f=f_{0}+3 f_{1}, \quad g=g_{0}+3 g_{1}
$$

be the canonical decomposition of the pair $(f, g)$. If $m_{1} \geq 5$ (see Definition 4) then by Theorem $4(\delta=2)$ there exists a primitive solution modulo 3 for 
the system

$$
f_{1} \equiv 0(\bmod 3), \quad g_{1} \equiv 0(\bmod 3),
$$

hence also a primitive solution modulo $3^{2}$ for the original system.

So we may assume $m_{1}<5$, and consequently $m_{0} \geq 17$, since $m_{0}+m_{1}=$ $n \geq 21$. According to Lemma 7 at least $\left[m_{0} / 3\right]-2$ new variables from the variables of the pair $\left(f_{0}, g_{0}\right)$ can be added at level 1 or higher. If one of these variables is at a level higher than one, then the solution used to produce this new variable is already a primitive solution modulo $3^{2}$. Therefore we may assume that all new variables coming from contraction are at level 1 . Our goal is to obtain $\widetilde{m}_{1} \geq 5$ (see introduction of Section 3 ) because this will ensure, by Theorem 4, a primitive solution modulo 3 for

$$
\widetilde{f}_{1} \equiv 0(\bmod 3), \quad \widetilde{g}_{1} \equiv 0(\bmod 3),
$$

hence also a primitive solution for the original system. And in fact,

$$
\widetilde{m}_{1} \geq m_{1}+\left[m_{0} / 3\right]-2 .
$$

Now it is easy to verify that in all cases given by the inequalities

$$
0 \leq m_{1}<5 \quad \text { and } \quad m_{0} \geq 21-m_{1}
$$

we will have $\widetilde{m}_{1} \geq 5$.

We now obtain a general condition, i.e., modulo $3^{t}$.

Lemma 9. Let $(f, g)$ be a pair of additive forms of degree $k=2 \cdot 3^{\tau} k_{0}$, with $\tau \geq 1,\left(k_{0}, 3\right)=1$. Then for any $t \geq 1$ the system of congruences

$$
\left\{\begin{array}{l}
f=a_{1} x_{1}^{k}+\ldots+a_{n} x_{n}^{k} \equiv 0\left(\bmod 3^{t}\right), \\
g=b_{1} x_{1}^{k}+\ldots+b_{n} x_{n}^{k} \equiv 0\left(\bmod 3^{t}\right)
\end{array}\right.
$$

admits a primitive solution modulo $3^{t}$ provided that $n \geq 2 \cdot 3^{t}+2 \cdot 3^{t-1}-3$.

Proof. We use induction on $t$. Notice that for $t=1,2$ the assertion is true by Theorem 4 and Lemma 8 .

Assume now that $t \geq 2$ and that every system of the form

$$
\left\{\begin{array}{l}
a_{1} x_{1}^{k}+\ldots+a_{n} x_{n}^{k} \equiv 0\left(\bmod 3^{t}\right), \\
b_{1} x_{1}^{k}+\ldots+b_{n} x_{n}^{k} \equiv 0\left(\bmod 3^{t}\right)
\end{array}\right.
$$

admits a primitive solution modulo $3^{t}$ if $n \geq 2 \cdot 3^{t}+2 \cdot 3^{t-1}-3$.

Let $n \geq 2 \cdot 3^{t+1}+2 \cdot 3^{t}-3$ and consider a system

$$
\begin{aligned}
& f=f_{0}+3 f_{1}+\ldots+3^{t} f_{t} \equiv 0\left(\bmod 3^{t+1}\right), \\
& g=g_{0}+3 g_{1}+\ldots+3^{t} g_{t} \equiv 0\left(\bmod 3^{t+1}\right)
\end{aligned}
$$

where $f, g$ are forms of degree $k$, written in canonical form. 
If $m_{1}+\ldots+m_{t} \geq 2 \cdot 3^{t}+2 \cdot 3^{t-1}-3$ then we can apply the induction hypothesis to the system

$$
\left\{\begin{array}{l}
f_{1}+3 f_{2}+\ldots+3^{t-1} f_{t} \equiv 0\left(\bmod 3^{t}\right) \\
g_{1}+3 g_{2}+\ldots+3^{t-1} g_{t} \equiv 0\left(\bmod 3^{t}\right)
\end{array}\right.
$$

obtaining a primitive solution modulo $3^{t}$, and hence a primitive solution modulo $3^{t+1}$ for (4).

Assume now that $m_{1}+\ldots+m_{t} \leq 2 \cdot 3^{t}+2 \cdot 3^{t-1}-4$.

Our goal here is then to contract as many variables as we can from level 0 in order to obtain

$$
\widetilde{m}_{1}+\ldots+\widetilde{m}_{t} \geq 2 \cdot 3^{t}+2 \cdot 3^{t-1}-3,
$$

because then by the induction hypothesis we can find a primitive solution modulo $3^{t}$ for the system

$$
\left\{\begin{array}{l}
\widetilde{f}_{1}+3 \widetilde{f}_{2}+\ldots+3^{t-1} \widetilde{f}_{t} \equiv 0\left(\bmod 3^{t}\right) \\
\widetilde{g}_{1}+3 \widetilde{g}_{2}+\ldots+3^{t-1} \widetilde{g}_{t} \equiv 0\left(\bmod 3^{t}\right)
\end{array}\right.
$$

hence also for

$$
\left\{\begin{array}{l}
\widetilde{f}_{0}+3 \widetilde{f}_{1}+3^{2} \widetilde{f}_{2}+\ldots+3^{t} \widetilde{f}_{t} \equiv 0\left(\bmod 3^{t+1}\right), \\
\widetilde{g}_{0}+3 \widetilde{g}_{1}+3^{2} \widetilde{g}_{2}+\ldots+3^{t} \widetilde{g}_{t} \equiv 0\left(\bmod 3^{t+1}\right)
\end{array}\right.
$$

and for the original system. In order to get to it we consider many cases:

If $3 \leq m_{1}+\ldots+m_{t} \leq 7=2 \cdot 3+1$ then $m_{0} \geq 2 \cdot 3^{t+1}+2 \cdot 3^{t}-3-7=$ $2 \cdot 3^{t+1}+2 \cdot 3^{t}-3^{2}-1$, hence

$$
\left[m_{0} / 3\right]-2 \geq 2 \cdot 3^{t}+2 \cdot 3^{t-1}-3-1-2=2 \cdot 3^{t}+2 \cdot 3^{t-1}-2 \cdot 3,
$$

which means that at least $2 \cdot 3^{t}+2 \cdot 3^{t-1}-2 \cdot 3$ new variables can be obtained by contraction of the variables of $\left(f_{0}, g_{0}\right)$. Hence

$\widetilde{m}_{1}+\ldots+\widetilde{m}_{t} \geq m_{1}+\ldots+m_{t}+2 \cdot 3^{t}+2 \cdot 3^{t-1}-2 \cdot 3 \geq 2 \cdot 3^{t}+2 \cdot 3^{t-1}-3$ since $m_{1}+\ldots+m_{t} \geq 3$.

Analogous argument can be applied to the cases $1 \leq m_{1}+\ldots+m_{t} \leq 2$ and $m_{1}+\ldots+m_{t}=0$.

Finally, if $m_{1}+\ldots+m_{t} \geq 8=2 \cdot 3+2$, then there exists $i \in\{0, \ldots, t-2\}$ such that

$$
2 \cdot 3^{t-i-1}+2 \cdot 3^{t-i-2} \leq m_{1}+\ldots+m_{t} \leq 2 \cdot 3^{t-i}+2 \cdot 3^{t-i-1},
$$

since $t \geq 2$, and then

$$
m_{0} \geq 2 \cdot 3^{t+1}+2 \cdot 3^{t}-3-\left(2 \cdot 3^{t-i}+2 \cdot 3^{t-i-1}\right),
$$

which implies that

$$
\left[m_{0} / 3\right]-2 \geq 2 \cdot 3^{t}-2 \cdot 3^{t-1}-2 \cdot 3^{t-i-1}-2 \cdot 3^{t-i-2}-1-2 .
$$


Hence we obtain after contraction at least $2\left(3^{t}-3^{t-1}-3^{t-i-1}-3^{t-i-2}\right)-3$ new variables. Then, since $m_{1}+\ldots+m_{t} \geq 2\left(3^{t-i-1}+3^{t-i-2}\right)$, we have

$$
\begin{aligned}
\widetilde{m}_{1}+\ldots+\widetilde{m}_{t} & \geq m_{1}+\ldots+m_{t}+2\left(3^{t}-3^{t-1}-3^{t-i-1}-3^{t-i-2}\right)-3 \\
& \geq 2 \cdot 3^{t}-2 \cdot 3^{t-1}-3 .
\end{aligned}
$$

4. $p$-Normalization. The concept and process of $p$-normalization was introduced by Davenport and Lewis. In [DL2] they associate with a given pair of additive forms $(f, g)$ as in (1) a parameter

$$
\vartheta=\vartheta(f, g)=\prod_{i \neq j}\left(a_{i} b_{j}-a_{j} b_{i}\right)
$$

and show that in order to prove existence of nontrivial common $p$-adic zeros for pairs of forms of the type (1) it is enough to consider pairs which satisfy the additional hypothesis $\vartheta \neq 0$. After defining equivalent pairs and $p$ normalized pairs they show that given a pair of forms $(f, g)$ with $\vartheta \neq 0$ there exists a $p$-normalized pair $\left(f^{*}, g^{*}\right)$ equivalent to $(f, g)$ with coefficients in $\mathbb{Z}$ and which has a $p$-adic zero if and only if the original pair does. Thus, concerning the existence of nontrivial common $p$-adic zeros for pairs of forms, it is sufficient to consider $p$-normalized pairs.

Moreover, if $(f, g)$ is $p$-normalized then one can show that its canonical form (2) is such that $s=k-1$.

Definition 6. Let $(f, g)$ be $p$-normalized and written in its canonical form. For each $i \in\{0,1, \ldots, k-1\}$ define $q_{i}$ to be the minimum number of variables appearing in any form $\lambda f_{i}+\mu g_{i}$ with coefficients not divisible by $p$ where $\lambda, \mu$ are not both divisible by $p$.

Davenport and Lewis in [DL2] show that when $(f, g)$ is $p$-normalized then

$$
\begin{aligned}
m_{0} & \geq n / k, \\
q_{0} & \geq n /(2 k) .
\end{aligned}
$$

Lemma 10. Let $k$ be the degree of the p-normalized pair $(f, g)$ and $\delta=$ $(p-1, k)$. Define

$$
r=\min \left(\left[\frac{m_{0}}{2 \delta+1}\right],\left[\frac{q_{0}}{\delta+1}\right]\right) .
$$

Then there exist $r$ disjoint pairs of subforms $\left(f_{0}^{i}, g_{0}^{i}\right)$ of $\left(f_{0}, g_{0}\right)$ such that

$$
2 \delta+1 \leq m_{0}^{i} \leq 2 \delta+2,
$$

and

$$
q_{0}^{i} \geq \delta+1
$$

for $i=1, \ldots, r$, where $m_{0}^{i}$ and $q_{0}^{i}$ are defined analogously to Definitions 4 and 6 .

Proof. The proof of this lemma is found in [DL2]. 


\section{The main theorems}

5.1. 3-adic case

Theorem A. If $k=2 \cdot 3^{\tau} k_{0}$, where $\left(k_{0}, 3\right)=1, \tau \geq 1$ and $n \geq 8 k^{2}$, then (1) is solvable in the field of 3-adic numbers.

Proof. Assume that $(f, g)$ is 3-normalized and consider its canonical form. By Lemma 3 it suffices to form an $\mathrm{NS}^{\tau+1}$ set. From (5) and (6) it follows that

giving

$$
m_{0} \geq 8 k \text { and } q_{0} \geq 4 k
$$

$$
r=\min \left(\left[m_{0} / 5\right],\left[q_{0} / 3\right]\right) \geq \frac{4}{3} k .
$$

According to Lemma 10 (since $\delta=2$ ) one can find, among the variables of $\left(f_{0}, g_{0}\right), r$ disjoint pairs of subforms with

$$
m_{0}^{i} \geq 5 \quad \text { and } \quad q_{0}^{i} \geq 3 \quad \text { for } i=1, \ldots, r .
$$

Now we can apply Theorem 5 to find a nonsingular solution modulo 3 for each of these $r$ pairs of subforms, that is, we can form $r$ NS3 sets.

Each NS3 set gives rise by contraction to a new variable at level 1 or higher. If any of them is at level higher than $\tau$ the result follows. Hence we may assume that all those new $r$ variables are at most at level $\tau$. We then form a system with them:

$$
\begin{aligned}
& 3\left(A_{1} T_{1}^{k}+\ldots+A_{r} T_{r}^{k}\right) \equiv 0\left(\bmod 3^{\tau+1}\right), \\
& 3\left(B_{1} T_{1}^{k}+\ldots+B_{r} T_{r}^{k}\right) \equiv 0\left(\bmod 3^{\tau+1}\right) .
\end{aligned}
$$

By Lemma 9 this system has a primitive solution modulo $3^{\tau+1}$ since

$$
r \geq \frac{4}{3} k \geq 2 \cdot 3^{\tau}+2 \cdot 3^{\tau-1}-3 .
$$

Let us denote it by $\vec{\xi}=\left(\xi_{1}, \ldots, \xi_{r}\right)$.

Since multiplication of a nonsingular solution by a constant which is coprime to 3 preserves nonsingularity (remember that among $\xi_{1}, \ldots, \xi_{r}$ there is at least one $\xi_{j}$ which is noncongruent to zero modulo 3 ), we conclude that $\xi_{1}, \ldots, \xi_{r}$ multiplied by the coordinates of the nonsingular solutions of the $r$ NS3 sets, will form an NS3 ${ }^{\tau+1}$, as desired.

Proposition 11. Let $k=6 k_{0}$, where $\left(k_{0}, 3\right)=1$. If $n \geq 28 k+1$ then the pair $(f, g)$ has a common 3-adic zero.

Proof. Assume that $(f, g)$ is 3-normalized. By Lemma 3 it is enough to form an NS3 ${ }^{2}$ set. Since $n \geq 28 k+1$, we have (see (5) and (6))

$$
m_{0} \geq n / k \geq 29, \quad q_{0} \geq n /(2 k) \geq 15
$$

which implies $r \geq 5$. As in Theorem A, by Lemma 10 and Theorem 5, we can form 5 disjoint NS3 sets. Contracting each of them we will have a system 
with $r \geq 5$ variables at level 1 or higher. Using Theorem 4 and the same ideas as in the above proof, we can find an $\mathrm{NS}^{2}$ set.

\subsection{2-adic case}

Lemma 12. The system

$$
\begin{aligned}
\left(a_{1} x_{1}^{k}+\ldots+a_{m} x_{m}^{k}\right) & \equiv 0\left(\bmod 2^{2}\right), \\
\left(b_{1} x_{1}^{k}+\ldots+b_{m} x_{m}^{k}\right) & \equiv 0\left(\bmod 2^{2}\right)
\end{aligned}
$$

has a primitive solution modulo 4 provided $m \geq 7$.

Proof. This is Lemma 2.16 of [G2], but it is important to remark that the primitive solution given in Lemma 2.16 is composed only by zeros and ones, therefore this solution is independent of the value of the degree $k$.

Proposition 13. If $n \geq 2 k^{5 / 2}$, where $k=2 k_{0}$, and $k_{0}$ is odd and different from one, then (1) is solvable in $\mathbb{Q}_{2}$.

Proof. This proof follows the lines of the proof of Theorem A above. By Lemma 3 it is enough to form an NS2 $2^{3}$ set. Since $n \geq 2 k^{5 / 2}$ and $k \geq 6$ we have

$$
\begin{gathered}
m_{0} \geq 2 k^{3 / 2} \Rightarrow m_{0} \geq 30 \\
q_{0} \geq k^{3 / 2} \Rightarrow q_{0} \geq 15 .
\end{gathered}
$$

Therefore

$$
r=\min \left\{\left[m_{0} / 3\right],\left[q_{0} / 2\right]\right\} \geq 7,
$$

for $\delta=1$. Now using Lemma 10 and Theorem 5, we can form 7 NS2. After contracting all those sets, we consider a new pair of forms involving all those new variables with their coefficients, say

$$
\begin{aligned}
h & =2\left(A_{1} T_{1}^{k}+\ldots+A_{7} T_{7}^{k}\right), \\
l & =2\left(B_{1} T_{1}^{k}+\ldots+B_{7} T_{7}^{k}\right) .
\end{aligned}
$$

Notice that, if we prove that the pair $(h, l)$ admits a primitive solution modulo $2^{3}$ then since those variables come from NS2 sets, the original pair admits a nonsingular solution modulo $2^{3}$. Now, the pair $(h, l)$ admits a primitive solution mod $2^{3}$ if and only if the system

$$
\begin{aligned}
\left(A_{1} T_{1}^{k}+\ldots+A_{7} T_{7}^{k}\right) & \equiv 0\left(\bmod 2^{2}\right), \\
\left(B_{1} T_{1}^{k}+\ldots+B_{7} T_{7}^{k}\right) & \equiv 0\left(\bmod 2^{2}\right)
\end{aligned}
$$

admits a primitive solution mod $2^{2}$. Thus, we can use Lemma 12 to find this primitive solution concluding this proof. 
5.3. The case $k=5^{\tau} 2 k_{0}, \tau \geq 1,\left(k_{0}, 5\right)=1$

Lemma 14. Let $u_{1}, \ldots, u_{s}, v_{1}, \ldots, v_{s} \in \mathbb{Z}$ where at least one in each pair $u_{i}, v_{i}$ is not divisible by $p^{r}$. Let $k=p^{\tau} \delta k_{0},\left(k_{0}, p\right)=1$, and $\delta=(p-1, k)$. If $s \geq(2 \delta+1)^{r}$ then the system

$$
\begin{aligned}
H & =u_{1} x_{1}^{k}+\ldots+u_{s} x_{s}^{k} \equiv 0\left(\bmod p^{r}\right), \\
J & =v_{1} x_{1}^{k}+\ldots+v_{s} x_{s}^{k} \equiv 0\left(\bmod p^{r}\right)
\end{aligned}
$$

has a primitive solution modulo $p^{r}$.

Proof. We use induction on $r$. If $r=1$ the result follows from Theorem 4 . Assume that the result is true for $r=t \geq 1$; we prove it for $r=t+1$. Write $(H, J)$ as (canonical form)

$$
\begin{aligned}
H & =H_{0}+p H_{1}+\ldots+p^{t} H_{t} \equiv 0\left(\bmod p^{t+1}\right), \\
J & =J_{0}+p J_{1}+\ldots+p^{t} J_{t} \equiv 0\left(\bmod p^{t+1}\right),
\end{aligned}
$$

denoting by $m_{j}$ the number of variables present at level $j$ (Definition 4 ).

Now divide the pair $(H, J)$ into $2 \delta+1$ pairs of subforms $\left(H^{(i)}, J^{(i)}\right)$, each pair having $(2 \delta+1)^{t}$ variables. Writing each pair $\left(H^{(i)}, J^{(i)}\right)$ in its canonical form, and denoting by $m_{j}^{(i)}$ the number of variables in the pair $\left(H^{(i)}, J^{(i)}\right)$ present at level $j$ we have

$$
i=1, \ldots, 2 \delta+1, \quad j=0,1, \ldots, t \quad \text { and } \quad m_{j}=m_{j}^{(1)}+\ldots+m_{j}^{(2 \delta+1)} .
$$

If, for some $i, m_{t}^{(i)}=0$ then the pair $\left(H^{(i)}, J^{(i)}\right)$ has $(2 \delta+1)^{i}$ variables occurring at levels less than $t$. Hence, we can use the induction hypothesis and find a primitive solution modulo $p^{t}$ for $\left(H^{(i)}, J^{(i)}\right)$. After contracting it, we will have a new variable at level $t$ or higher. If the level is higher than $t$, we already have a primitive solution modulo $p^{t+1}$. So assume that all the new variables coming from contractions of all the pairs $\left(H^{(i)}, J^{(i)}\right)$ which satisfy $m_{t}^{(i)}=0$ are at level $t$. We then form a system with the variables which have already been at level $t$ (i.e., $m_{t}^{(i)} \neq 0$ ) together with those new ones, say,

$$
\begin{aligned}
p^{t}\left(A_{1} T_{1}^{k}+\ldots+A_{w} T_{w}^{k}\right) & \equiv 0\left(\bmod p^{t+1}\right), \\
p^{t}\left(B_{1} T_{1}^{k}+\ldots+B_{w} T_{w}^{k}\right) & \equiv 0\left(\bmod p^{t+1}\right),
\end{aligned}
$$

where at least one in each pair $A_{i}, B_{i}$ is not divisible by $p$. Since there are $2 \delta+1$ pairs of the form $\left(H^{(i)}, J^{(i)}\right)$, we have $w \geq 2 \delta+1$. We can then use Theorem 4 and conclude this proof.

Proposition 15. If $k=5^{\tau} 2 k_{0}, \tau \geq 1,\left(k_{0}, 2\right)=1=\left(k_{0}, 5\right)$ and $n \geq$ $2 k^{5 / 2}$, then the pair $(f, g)$ has a common 5 -adic zero. 
Pro of. By Lemma 3 it is enough to form an NS5 ${ }^{\tau+1}$ set. Since $n \geq 2 k^{5 / 2}$, we have

$$
\begin{aligned}
m_{0} \geq 2 k^{3 / 2} & \Rightarrow m_{0} \geq 2\left(5^{\tau} 2\right)^{3 / 2}, \\
q_{0} \geq k^{3 / 2} & \Rightarrow q_{0} \geq\left(5^{\tau} 2\right)^{3 / 2},
\end{aligned}
$$

therefore, since $\delta=(k, 4)=2$,

$$
r=\min \left\{\left[m_{0} / 5\right],\left[q_{0} / 3\right]\right\} \geq 5^{\tau} .
$$

Now, using Lemma 10 and Theorem 5 , we can form $5^{\tau}$ NS5 sets, and, after contraction and assuming that all those new variables lie at level less than $\tau+1$, we can form a system with all those new variables which will look like

$$
\begin{aligned}
& 5\left(A_{1} T_{1}^{k}+\ldots+A_{s} T_{s}^{k}\right) \equiv 0\left(\bmod 5^{\tau+1}\right), \\
& 5\left(B_{1} T_{1}^{k}+\ldots+B_{s} T_{s}^{k}\right) \equiv 0\left(\bmod 5^{\tau+1}\right),
\end{aligned}
$$

where $s=5^{\tau}$. But then $s=(2 \delta+1)^{\tau}$. Applying Lemma 14 we conclude that this system has a primitive solution, which guarantees that therefore an $\mathrm{NS} 5^{\tau+1}$ set exists in the original pair.

\subsection{Proof of Theorem $B$}

Theorem B. If $n \geq 2 k^{5 / 2}$ then (1) is solvable in $\mathbb{Q}_{p}$ for every prime $p$, provided $k \neq 2^{2}, 2^{3}, 2^{4}, 2^{5}$.

As already remarked in the introduction, we need only pay attention to the degrees of the form $k=p^{\tau}(p-1) k_{0}$ with $\tau \geq 1$ and $\left(k_{0}, p\right)=1$, and the particular case $k=5^{\tau} 2 k_{0}, \tau \geq 1,\left(k_{0}, 5\right)=1$. For all other cases the conjecture of Artin is true, as proved in [DL2].

The case $k=5^{\tau} 2 k_{0}, \tau \geq 1,\left(k_{0}, 5\right)=1=\left(k_{0}, 2\right)$ is treated in Proposition 15. If $k=5^{\tau} 2 k_{0}, \tau \geq 1,\left(k_{0}, 5\right)=1$ but $\left(k_{0}, 2\right)=2$ then $k$ is of the form $k=p^{\tau}(p-1) k_{0}$ with $\tau \geq 1$ and $\left(k_{0}, p\right)=1$. Hence from now on we assume $k=p^{\tau}(p-1) k_{0}$ with $\tau \geq 1$ and $\left(k_{0}, p\right)=1$. In this case:

If $p \geq 5$ then we assert that the pair $(f, g)$ has a common $p$-adic zero. In fact, from Theorem 2 we know that the pair $(f, g)$ has a common $p$-adic zero provided $n \geq 2 k^{2+w(\tau, p)}$, and it is simple to verify that

$$
p \geq 5 \Rightarrow w(\tau, p) \leq 1 / 2 \text {. }
$$

Hence we conclude that $n \geq 2 k^{5 / 2}$ implies $n \geq 2 k^{2+w(\tau, p)}$ and (1) is solvable in $\mathbb{Q}_{p}$.

If $p=3$ then $k=3^{\tau} 2 k_{0}$, and in this case

$$
2 k^{5 / 2} \geq 8 k^{2}
$$

unless $\tau=1$ and $k_{0} \leq 2$. From Theorem A we conclude that unless $\tau=1$ and $k_{0} \leq 2$ the pair $(f, g)$ is solvable in $\mathbb{Q}_{3}$. And if $\tau=1$ and $k_{0} \leq 2$, i.e., $k=6$ or $k=12$ we use Proposition 11 to guarantee the solvability of (1) in 
$\mathbb{Q}_{3}$ since

$$
2 k^{5 / 2} \geq 28 k+1 \text {. }
$$

Finally if $p=2$ then $k=2^{\tau} k_{0}, k_{0}$ odd. The case $k=2$ is treated in [D] (see introduction) and the case $k=2 k_{0}$ with $k_{0}$ odd and $k_{0} \neq 1$ is treated in Proposition 13. Hence we are left with the case

$$
k=2^{\tau} k_{0}, \quad \tau \geq 2, \quad k_{0} \text { odd }
$$

By Lemma 3, we want to guarantee the existence of an $\mathrm{NS}^{\tau+2}$ set.

Observe now that, since for any $x$ and for any $\tau \geq 2$,

$$
x^{2^{\tau}} \equiv 0 \text { or } 1\left(\bmod 2^{\tau+2}\right)
$$

(proof by induction on $\tau$ ), we have for any $x$ and for any $\tau \geq 2$,

$$
x^{2^{\tau} k_{0}} \equiv x^{2^{\tau}}\left(\bmod 2^{\tau+2}\right) .
$$

Hence any nonsingular solution modulo $2^{\tau+2}$ for pairs of degree $k=2^{\tau}$, $\tau \geq 2$, will also be a nonsingular solution for the pairs involving the same coefficients but of degree $k=2^{\tau} k_{0}$ for any $k_{0}$. And since

$$
2\left(2^{\tau}\right)^{5 / 2} \geq 16\left(2^{\tau}\right)^{2}-26\left(2^{\tau}\right)+1,
$$

except for $\tau=2,3,4,5$, we could use Theorem 1 to guarantee a common 2 -adic zero for the pair of degree $2^{\tau}$, with the possible exception for $k=$ $2^{2}, 2^{3}, 2^{4}, 2^{5}$.

The case of degree $2^{2}=4$ was treated in [G1], where it is proved that pairs of additive quartic forms have common 2-adic zeros provided the number of variables exceeds $60\left(<2(4)^{5 / 2}\right)$.

We conclude this paper with the following result, a straightforward consequence of which we have seen so far. This gives a better understanding of the results we have obtained, and makes it clear that the correct order of magnitude for the number of variables is smaller than $2 k^{3}$.

Theorem C. Let $(f, g)$ be a pair of additive forms of degree $k$ in $n$ variables. Then $(f, g)$ has a common p-adic zero for every prime $p$, whenever one of the following cases holds:

1. $n \geq 2 k^{2.5}$ and $k \neq 2^{3}, 2^{4}, 2^{5}$;

2. $n \geq 2 k^{2.59}$ and $k \neq 2^{3}, 2^{4}$;

3. $n \geq 2 k^{2.72}$ and $k \neq 2^{3}$;

4. $n \geq 2 k^{2.9}$.

Proof. The first assertion is Theorem B together with the case $k=2^{2}$ treated in [G1]. For all the other cases, it is easy to verify that, taking $s=2.59,2.72$ and 2.9 for $k=2^{5}, 2^{4}$ or $2^{3}$ respectively, one has

$$
2 k^{s} \geq 16 k^{2}-26 k+1 \text {, }
$$


which ensures that, if $n \geq 2 k^{s}$, then (1) is solvable in $\mathbb{Q}_{p}$ for all primes $p$ by Theorem 1.

\section{References}

[AC] O. D. Atkinson and R. J. Cook, Pairs of additive congruences to a large prime modulus, J. Austral. Math. Soc. Ser. A 46 (1989), 438-455.

$[\mathrm{ABC}]$ O. D. Atkinson, J. Brüdern and R. J. Cook, Simultaneous additive congruences to a large prime modulus, Mathematika 39 (1992), 1-9.

[DL1] H. Daven port and D. J. Lewis, Cubic equations of additive type, Philos. Trans. Roy. Soc. London Ser. A 261 (1966), 97-136.

[DL2] -, - Two additive equations, Proc. Sympos. Pure Math. 12 (1967), 74-98.

[D] V. B. Demyanov, Pairs of quadratic forms over a complete field with discrete norm with finite residue class field, Izv. Akad. Nauk SSSR 20 (1956), 307-324 (in Russian).

[G1] H. Godinho, A pair of additive quartic forms, Ph.D. thesis, University of Michigan, 1992.

[G2] -, Additive forms of degree $k=2^{l}$, J. Number Theory 46 (1994), 391-408.

[G3] -, On p-adic zeros of additive forms of even degree, ibid., to appear.

[W] T. D. Wooley, On simultaneous additive equations II, J. Reine Angew. Math. 419 (1991), 141-198.

Departamento de Matemática

Instituto de Matemática

UnB

UFRS

Brasília, DF, Brasil, 70910-900

E-mail: hemar@mat.unb.br

Porto Alegre, RS, Brasil, 91509-900

E-mail: cydara@if.ufrgs.br

Received on 28.7.1997

and in revised form on 30.3.1999 